\title{
CAMBIOS CICLICOS Y TENDENCIAS A LARGO PLAZO EN LA SALINIZACIÓN DE ECOSISTEMAS FLUCTUANTES (ALBUFERAS DE ADRA). EVIDENCIAS DE EUTROFIZACIÓN Y CONTAMINACIÓN.
}

\author{
P. Carrillo; P. Sánchez Castillo*; L. Cruz Pizarro y R. Morales \\ Departamento de Biologia Animal, y Ecologia. Univ. de Granada. \\ * Departamento de Biologia Vegetal. Univ. de Granada.
}

Palabras clave: Cambios ciclicos. Tendencias. Salinización. Estratificación. Contaminación. Albuferas de Adra.

Key words: Seasonal vs long-temchanges. Salinization. Contamination. Adra costal lagoons.

\begin{abstract}
SEASONAL CHANGES AND LONG-TERM TENDENCIES ON THE SALINIZATION OF FLUCTUATING ECOSYSTEMS (ADRA COASTAL LAGOONS). EVIDENTES OF EUTROPHICATION AND CONTAMINATION.

A seasonal study on the chemical and physical parameters carried out at the point of maximun depth along the vertical profile in two lagoons from the Albufera of Adra (Almeria) has allowed us to characterize them as eutrophic, with basic waters well oxygenated which maintain high levels of alkalinity and mineralization. Their ionic proportions being closer to marine than to freshwaters.

Vertical distribution is quite uniform for most of the parameters along the annual cycle and it is difficult to visualize a well defined cyclic changes. On the basis of this study and from comparisons with previous data on nutrients and conductivity, it can be shown an intense eutrophication rhythm, and the variations on the rates $\mathrm{Cl} / \mathrm{SO}_{4}{ }^{-} \mathrm{y} \mathrm{Ca}^{++}+\mathrm{Mg}^{++} / \mathrm{Na}^{++} \mathrm{K}^{+}$seems to define a "marine" period during the summer alterning with a "fresh" one along the winter.
\end{abstract}

\section{INTRODUCCIÓN}

De entre los medios acuáticos litorales, las Albuferas son uno de los más importantes. Constituyen reservorios de agua "dulce" que por su situación están sometidos a influencias marinas y continentales.

Por lo común se situan en zonas de fuerte presión derivadas de actividades agrícolas e industriales que les hace ser ecosistemas fluctuantes con cierto grado de imprevisibilidad (MIRACLE. 1987).

Estas razones, junto con su amplia distribución geográfica y el hecho de constituir áreas de importantes recursos alimen- ticios ha despertado, desde antiguo, un gran interés por su estudio.

La Albufera de Adra, se sitúa en el extremo oriental del delta del rio Adra, constituyendo un sistema lagunar que, en la actualidad, está formado por cuatro lagunas de diferente tamaño (CARRILLO et al., 1988) rodeadas casi por completo por terrenos dedicados a la agricultura intensiva (cultivos forzados de invernadero).

En este trabajo se presentan los resultados del análisis de algunas caracteristicas fisicas y quimicas de las aguas de las dos mayores (Honda: 1.6 Ha; Prof. max. $1.5 \mathrm{~m}$, y Nueva: $3 \mathrm{Ha}$; Prof. max. $2 \mathrm{~m}$ ) que se llevó a cabo durante un ciclo anual 
(Diciembre de 1984 a Enero de 1986) y que es parte de un estudio mas amplio subvencionado por el Instituto de Estudios Almerienses, cuyo objetivo básico era la caracterización ecológica de dichos ecosistemas y la cuantificación de los efectos derivados de las actividades humanas en los últimos años.

Los resultados correspondientes a los cambios estacionales de las comunidades planctónicas así como de los parámetros biológicos relacionados (clorofila a, indice de pigmentos, diversidad, sucesión...) se muestran en CARRILLO er al. (1988) y SÁNCHEZ-CASTILLO et al. (1987), además DEL RÍO (1986) estudia los procesos de contaminación por plaguicidas procedentes de los cultivos que las rodean.

\section{MATERIAL Y MÉTODOS}

Los muestreos se realizaron con periodicidad aproximadamente mensual en la zona de máxima profundided de cada una de las lagunas, en un ambiente de caracteristicas esencialmente limnéticas, entre las 10 y las $12 \mathrm{~h}$ solares.

Una boya señaló la posición de ambas estaciones durante el ciclo anual de estudio.

"In situ" se midieron los siguientes parámetros: Transparencia, a través de la profundidad de visión del disco de Secchi (un disco metálico blanco de $20 \mathrm{~cm}$ de diámetro), Temperatura, $\mathrm{pH}$ y Conductividad de cada una de las profundidades muestreadas, utilizando un autoanalizador ambiental "Hydrolab" (mod. 4041) con sonda de profundidad.
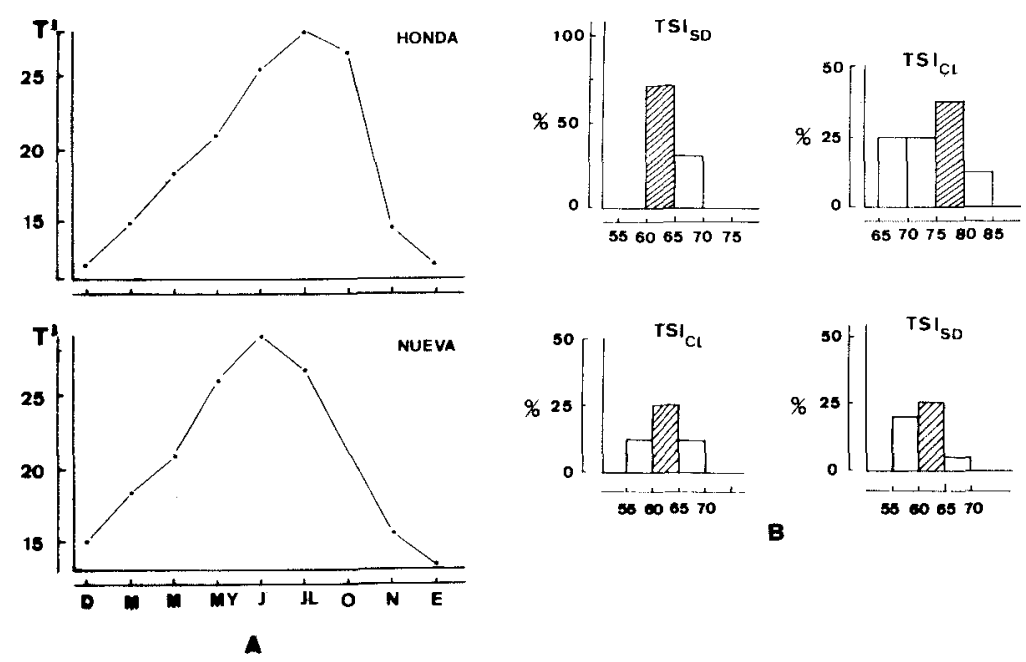

Figura 1.- A) Evolución de la temperatura media en el perfil vertical de ambas laguna B) Frecuencias de los valores TSI (SD) y TSI $_{(\mathrm{Cla})}$

E1 agua para las determinaciones analíticas se recogió con una doble botella de Van Dom de 8 I de capacidad cada una, sujeta a un cable graduado a intervalos de $25 \mathrm{~cm}$, que se sumergía $\mathrm{cn}$ cl perfi] vertical a intervalos regulares de $0.5 \mathrm{~m}$ de profundidad. De este volumen se tomaron, con las precauciones que exige el método empleado (Winkler, modificado de acuerdo con GOLTERMAN (1969)), $300 \mathrm{ml}$ en botellas de vidrio opacas de tipo BOD para la determinación del Oxigeno disuelto. Las muestras eran fijadas inmediatamenteañadiendo $1 \mathrm{ml}$ de $\mathrm{SO}_{4} \mathrm{Mn}$ y $1 \mathrm{ml}$ de $\mathrm{IK}-\mathrm{KOH}$.

Las muestras destinadas a los análisis de Alcalinidad. compuestos inorgánicos de Nitrógeno y Cloruros se filtraron a través un filtro Whatman GF/C y fueron fijadas con cloroformo. Así se transportaban congeladas al laboratorio.

Para su determinacion se siguió la metodologia habitualmente empleada en estudios limnológicos y de agua marina (APHA, 1976; ROS, 1979).

\section{RESULTADOS Y DISCUSION}

\section{Características físicas y nivel de eutrofia}

Durante el ciclo anual de estudio no se han observado indicios de establecimiento de termoclina en ninguna de las dos lagunas estudiadas. Las diferencias superficie-fondo apenas llegan a ser de 0.5 " $\mathrm{C}$ en la laguna Honda y ligeramen te superiores a $1.5^{\circ} \mathrm{C}$ en la Nueva. El régimen de vientos en la zona y la poca profundidad de las lagunas son, probablemte, los responsables del mantenimientode una mezcla prolongada de las aguas.

En la figura la se muestra la evolución de la temperatura media de la columna de agua a lo largo del periodo de estudio. 
El patrón de variación es muy similar en ambas lagunas, con un periodo de calentamiento que se inicia a final de Enero y continua hasta final de Septiembre y otro, mas corto, de enfriarniento desde Octubre a Enero.

De la medida de la profundidad de visión del disco de Secchi se desprende que, en ambas lagunas, el espesor de la zona eufótica (cuyo límite se estima como la profundidad a la que se reduce a 11100 la luz incidente en superficie) llegaría hasta el fondo de las mismas, comprendiendo todo el volumen.

En la figura $I b$ se ha representado la distribución de frecuencias de los valores del índice de estado trófico (TSI) calculado siguiendo a SHAPIRO et al. (1975) a partir de los datos de visión del disco de Secchi (TSISD) Y de clorofila a (TSICla). Ambas lagunas presentan un elevado nivel de eutrofia siendo, en general, la laguna Honda más eutrófica que la Nueva, diferencia que es particularmente evidente a partir de los datos de clorofila a.

E1 hecho de que en ocasiones, y dentro de una misma laguna, se encuentren diferencias en las estimas del TSI a partir de los valores de transparencia y de clorofila a significa, seguramente, que la proliferación de algas planctónicas no es siempre la única causa que limita la penetración de la luz. La materia inorgánica en suspensión, favorecida por los fuertes vientos de la zona jugará, así mismo un papel importante (DEL RÍO, 1986).

\section{Características químicas.}

Los valores de Oxigeno disuelto medidos son bastante elevados y suponen niveles próximos a la saturación en buena parte del ciclo anual. Concretamente en la laguna Honda se han medido valores comprendidos entre $4.4 \mathrm{mg} / \mathrm{l}$ el dia $1 \mathrm{ll}-\mathrm{V}$ 85, en las proximidades del fondo, y $12 \mathrm{mgll}$, en superficie el dia 3-XI-85. Los valores extremos registrados en la laguna Nueva, más oxigenada en promedio, fueron de $4.8 \mathrm{mg} / \mathrm{l}$ en superficie, el 30-111-85 y $11.2 \mathrm{mg} / 1$ durante el muestreo correspondiente al 9-111-85 en las proximidades del sedimento.

La distribución en el perfil vertical muestra una cierta uniformidad en ambos sistemas (SÁNCHEZ CASTIILLO er $a l ., 1987)$ y aunque es difícil establecer un patron definido en la laguna Honda parecen evidenciarse máximos superficiales (concomitantes con mínimos en la proximidad del fondo) durante la época de invierno que, a lo largo de la primavera y el verano, se van desplazando hacia los 0.5 y 1.0 metros de
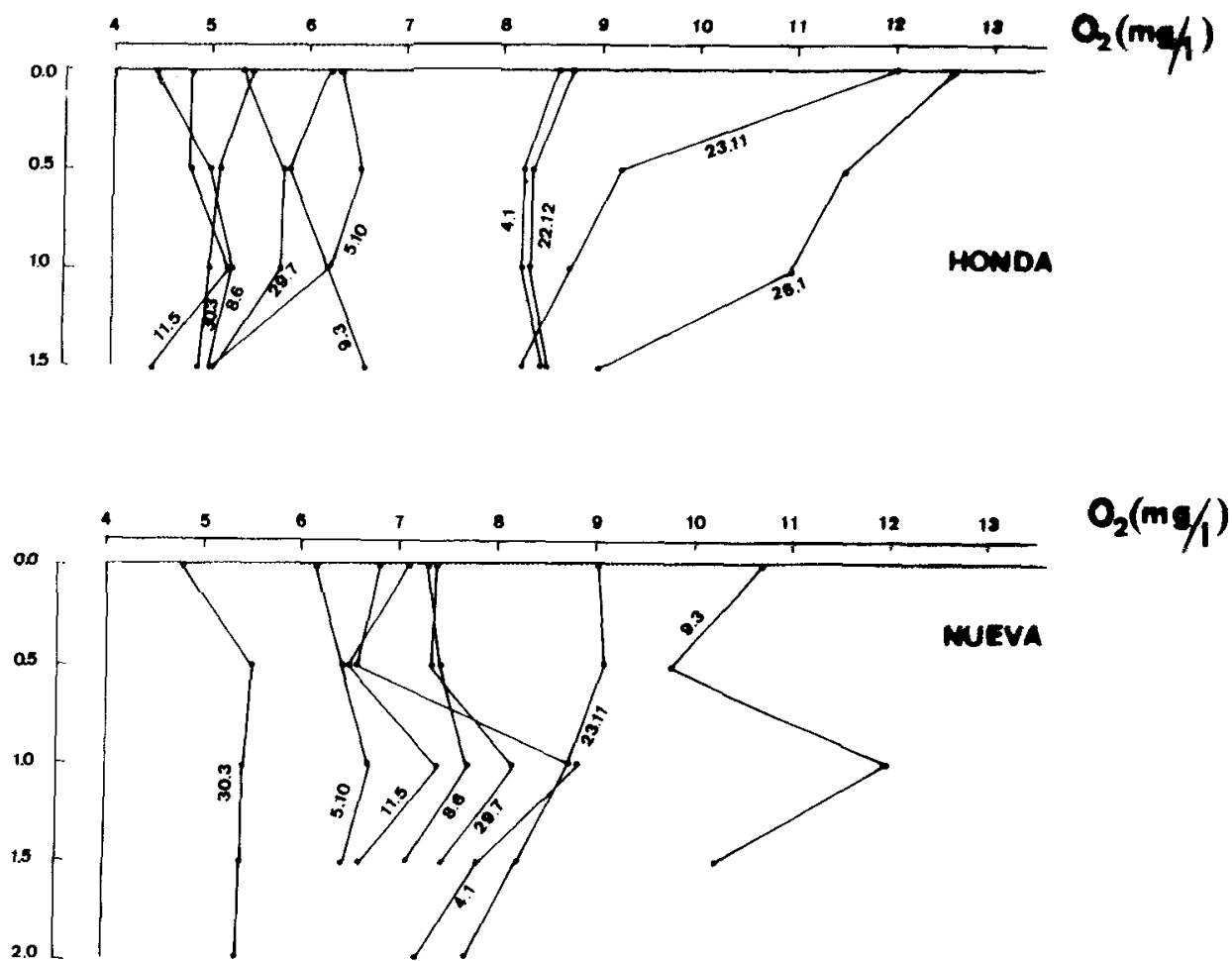

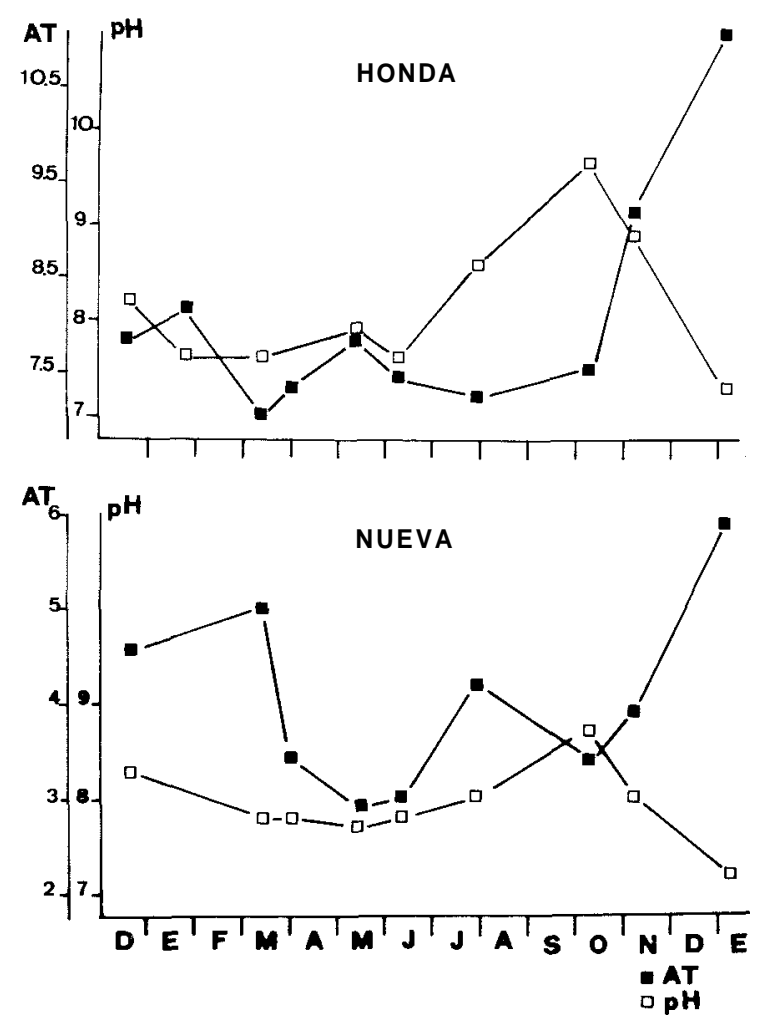

FIGUKA 3.Variación de los valores medios de Alcalinidad (meg $/ /^{11}$ ) y $\mathrm{pH}$ en el perfil vertical de las lagunas estudiadas.

profundidad. En la laguna Nueva los máximos en profundidad se situan a $1.0 \mathrm{~m}$. durante, practicamente, todo el ciclo anual (Fig. 2).

La evolución de los valores promedio en el perfil vertical a lo largo del año sigue un patrón en esencia similar en las dos lagunas en el que destacan maximos invernales de corta duración junto a valores menores y poco fluctuantes durante el rcsto del año. Esto es particularmente evidente en la laguna Honda en la que la correlación negativa altamen te significativa entre oxigeno y temperatura (Fig. 7) parece sugerir que dicha evolución está regida, básicamente, por procesos físicos (incremento de solubilidad) y en la que debe intervenir decididamemte la agitación turbulenta del agua provocada por el viento.

Ambas lagunas presentan aguas básicas. No se aprecia una variacidn definida del $\mathrm{pH}$ en profundidad y son mínimas las diferencias que se observan en la evolución de los valores medios en el perfil vertical durante buena parte del ciclo anual, siendo únicamente destacables las elevaciones que se observan hacia el final del verano, particularmente en la laguna Honda (Fig. 3).

Las elevadas concentraciones de carbonatos y bicarbonatos determinan una reserva alcalina considerable. Son, igualmcnte, poco apreciables las variaciones en profundidad y a partir de los datos de que disponemos no resulta fácil explicar los máximos invcrnales de alcalinidad total a los que probablemente contribuyan procesos de redisolución de carbonatos - concomitantes con máximos de fosfatos disueltos (DEL RÍO, 1986) - y un aporte de origen fluvial.

Se han medido concentraciones muy elevadas de nitrogeno inorgánico si bien las concentraciones relativas de las distintas formas analizadas así como sus distsibuciones espaciotemporales parecen ser un buen ejemplo de la Influencia de procesos externos al metabolismo de ambos sistemas, en las caracteristicas de las mismas.

En la laguna Honda, la de mayor nivel de eutrofia, el nitrato es la forma predominante, aunque son las concentraciones de nitrito y de amonio las que presentan mayor amplitud de variacidn (Fig. 4). Los correspondientes rangos de variacidn de
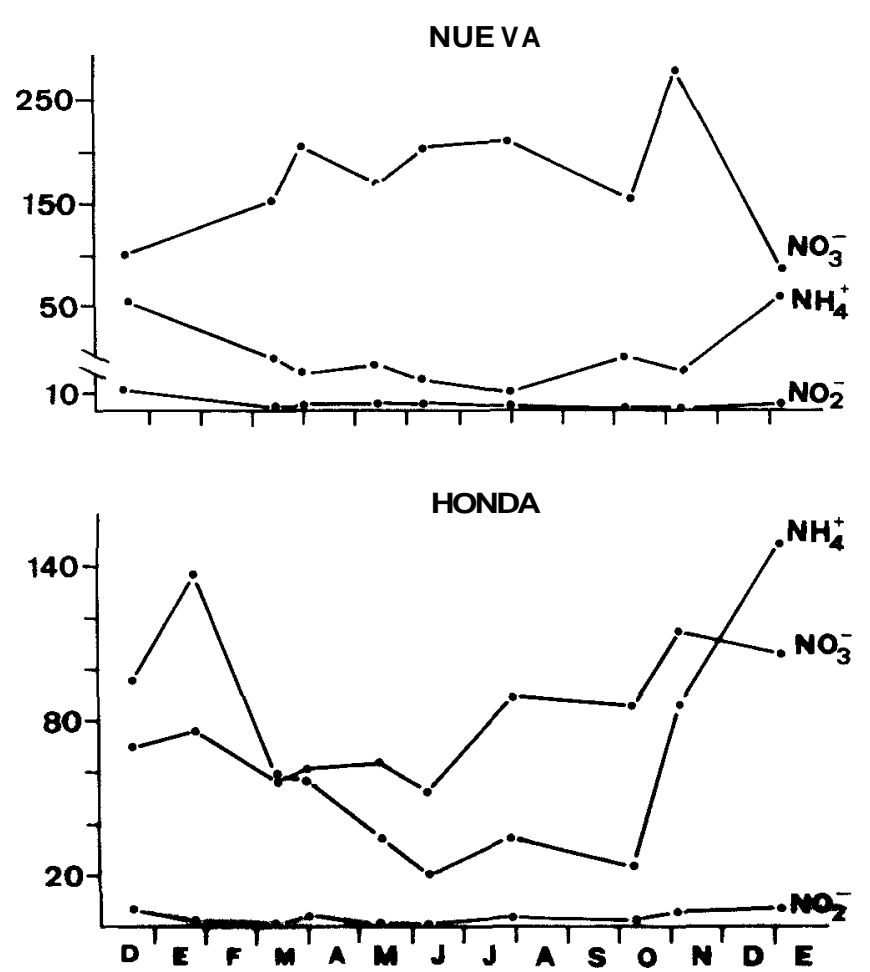

FIGURA 4. Concentraciones de nitrógeno inorgánico $\left(\mathrm{mg}-\mathrm{at} \mathrm{N} \cdot \mathrm{f}^{-1}\right)$ a lo largo de un ciclo anual en ambas lagunas. 


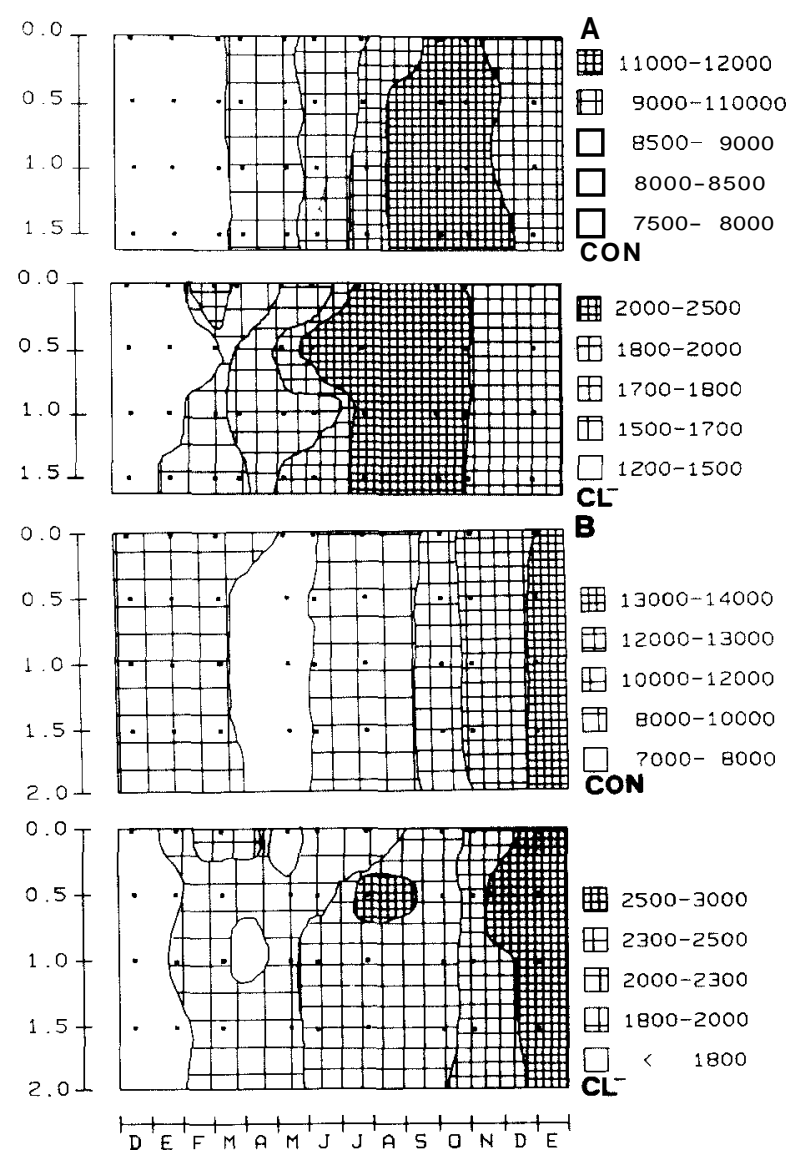

FIGURA 5. Distribución espacio-temporal de la conductividad $(\mu \mathrm{S} \cdot \mathrm{cm})$ y Cloruros $\left(\left.\mu \mathrm{g}\right|^{\prime}\right)$. A) Laguna Honda. B) Laguna nueva.

las diferentes fracciones en la laguna Nueva son considerablemente más amplios.

Aunque en líneas generales las variaciones en el perfil vertical son mínimas en ambas lagunas, en la Honda parece desarrollarse un máximo estival de nitratos que se sitúa en las capas mas profundas mientras que los máximos de OtoñoInvierno y los minimos de Primavera-Verano que se observan para los nitritos y el amonio se distribuyen uniformemente en profundidad (SÁNCHEZ CASTILLO et al., 1987).

Para tratar de explicar la situación que se observa en esta laguna así como las correlaciones que se estableccn entre las distintas formas de este nutriente con el oxígeno y la temperatura tal vez hubiera que pensar en una contribución de los ecosistemas adyacentes a las lagunas (campos de cultivo) a la concentración de nitratos de las aguas a través de perdidas por lixiviación. como apunta BREZONIK (1972).
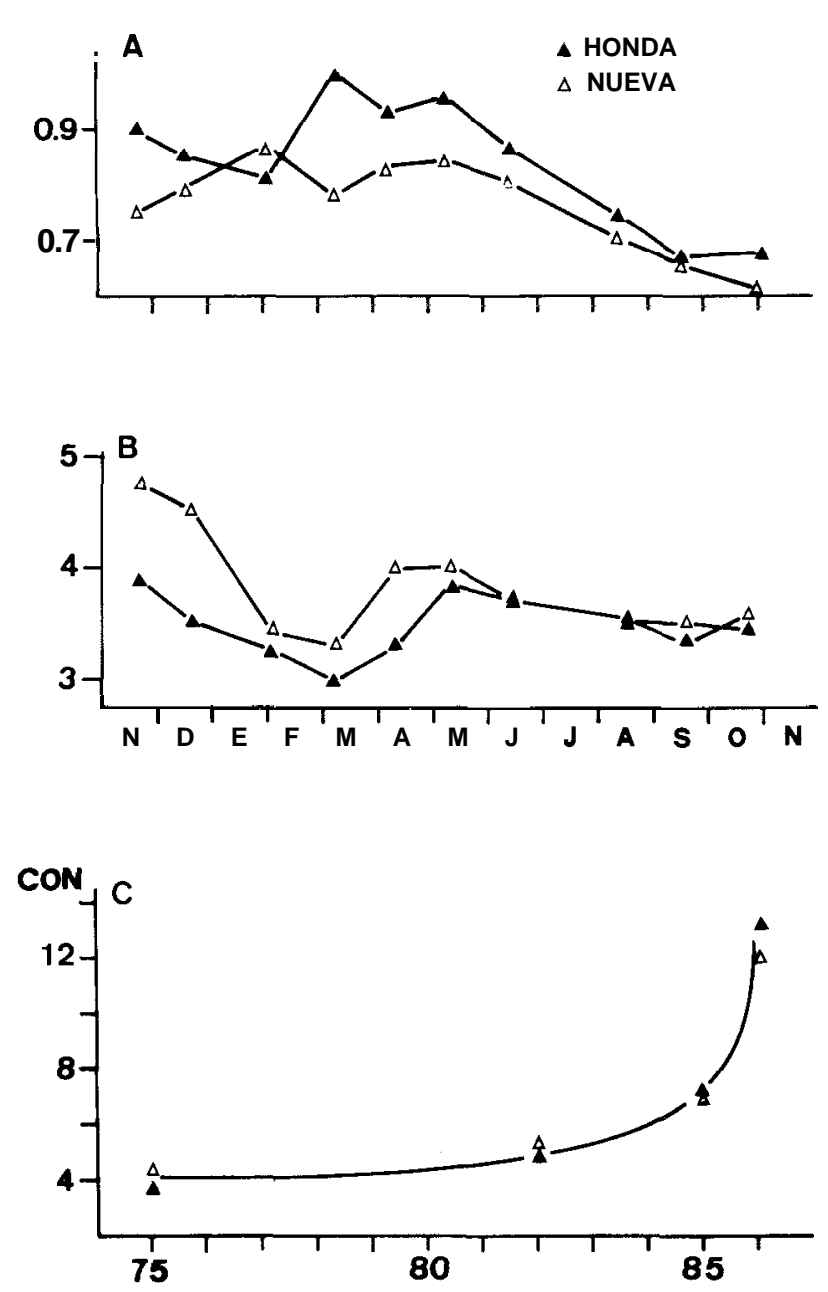

FIGURA 6 Valores de las relaciones $\mathrm{Ca}^{++}+\mathrm{Mg}^{+} / \mathrm{Na}^{+}+\mathrm{K}^{\prime}$ (A) y $\mathrm{Cl} / \mathrm{SO}^{-}{ }_{4}$ (B) en ambas lagunas a lo largo del tiempo, e incremento en los valores de condoctividad $\left(\mathrm{mS} \cdot \mathrm{cm}^{1}\right)(\mathrm{C})$ en las dos lagunas (varios autores).

En la laguna Nueva, los máximos de las distiritas fracciones estan mas separadas en el tiempo (Fig. 4) y sobre todo en el espacio (SÁNCHEZ CASTILLO et al., 1987).

Ambas lagunas presentan aguas fuertemente mineralizadas 'con valores de conductividad comprendidos entre 7.5 y 11.9 $\mathrm{mS} \cdot \mathrm{cm}^{1}$ en la laguna Honda y entre 7.5 y $13.2 \mathrm{mS} \cdot \mathrm{cm}^{1}$ en la Nueva.

La semejanza entre ambas lagunas se extiende, asimismo a la uniformidad que se observa en la distribución dentro del perfil vertical y, en menor proporción, en relación con cl patrón de evolución temporal que, básicamente, se caracteriza por presentar los mayores valores de mineralización en Verano y Otoño, coincidiendo con una variación casi paralela de los cloruros (Fig. 5). De hecho la correlacion entre ambos 

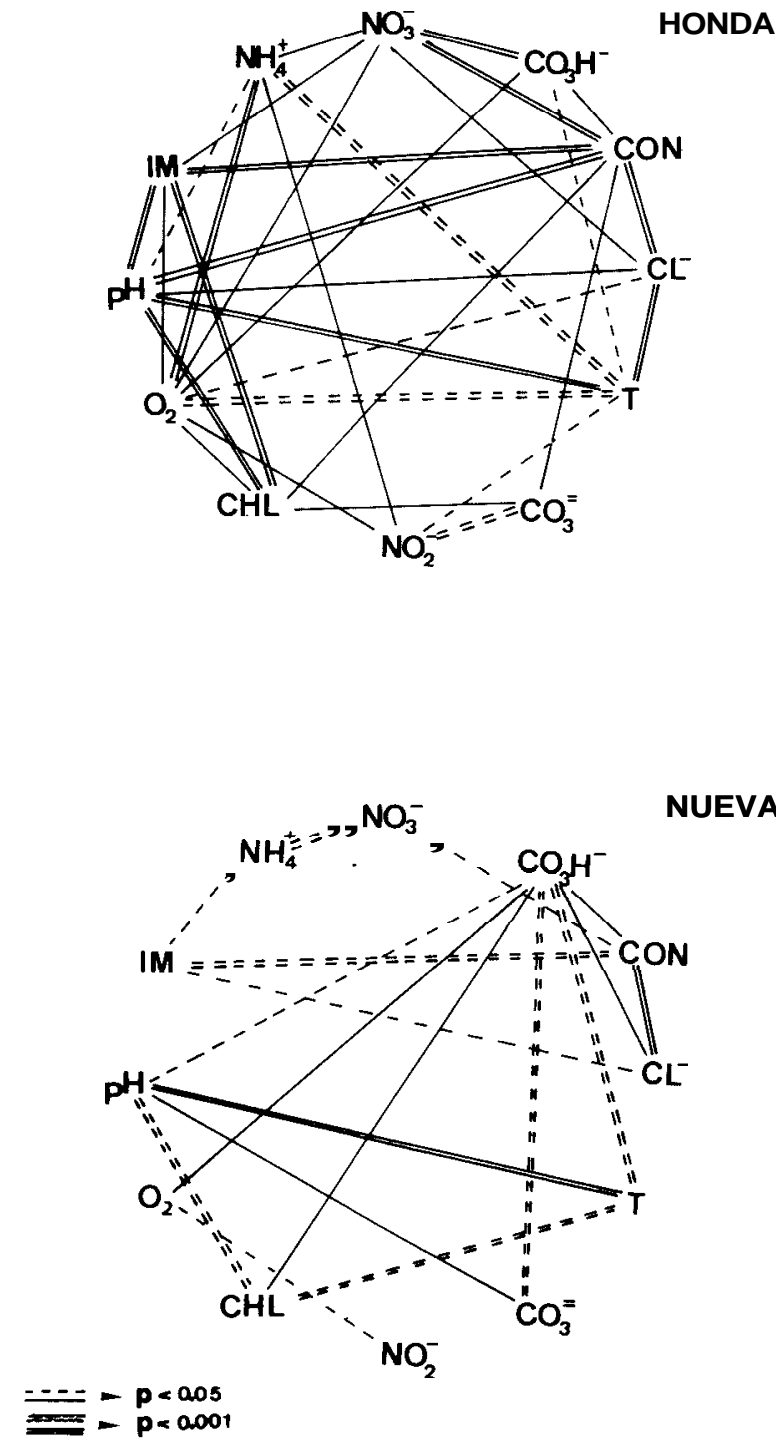

FIGURA 7. Correlaciones entre los parámetros medios en nmbas lagunas. (-) corselacion positiva (---) correlacion negativa.

parametros es positiva y altamente significativa en ambas lagunas (Fig. 7).

En estos parámetros, como en la mayoria de los estudiados, resulta difícil visualizar un carácter cíclico en su variación anual y, los resultados obtenidos en años sucesivos muestran, asimismo, diferencias notables. Tal el es el caso de la evolucion creciente de los valores de conductividad que se observa sobre datos de 11 años (Fig. 6c), o los que se derivan de la comparacion de los valores de nutrientes obtenidos en este trabajo con los que se citan en IGME (1975) y LOPEZ (1983).

En última instancia estas consideraciones evidenclan la importancia fundamental de la influencia humana (actividades derivadas de prácticas agrícolas, contaminación, etc.) que se superpone a las particularidades hidrológicas de las lagunas y a los procesos físico-químicos y biológicos que tienen lugar en su interior.

Este "stress" da lugar a fluctuaciones amplias en los distintos parámetros y, posiblemente, como ocurre en la Albufera de Valencia (SERRA et al., 1984) el ritmo de eutrofizacidn es lo suficientemente intenso para quedar plasmado en cambios no sólo cíclicos sino tendenciales sobre periodos de tiempo superiores a un año.

La falta de cuantificación de la influencia de aquellas actividades sobre las aguas de las lagunas, así como el desconocimiento casi general de la hidrologia particular (régimen hidrico) de la Albufera, han determinado que los intentos de relacionar las variaciones en la composición quimica de las aguas con las características climatológicas (SÁNCHEZ CASTILLO, 1984) o con procesos derivados de la alteración en el aporte de agua procedente del acuifero e intrusiones marinas (DEL RÍO, 1986) no hayan sido satisfactorias.

Tan solo la evolución de la relación molar $\left(\mathrm{Ca}^{++}+\mathrm{Mg}^{++}\right) /\left(\mathrm{Na}^{+}\right.$ $+\mathrm{K}$ ) (Fig. 6) y de la relación $\mathrm{Cl} / \mathrm{SO} 4$, que hemos calculado de los datos de DEL RíO (1986), parecen mostrar un patrón que pudiera hacer sospechar intrusiones marinas durante la etapa de estiaje (particularmente en la laguna Nueva), que alternan con periodos de mayor influencia del agua dulce (especialmente patentes en la laguna Honda). Si bien los valores de dichas relaciones siempre han estado mucho más próximos a los del agua marina, un carácter que queda reforzado por la relación de las proporciones iónicas, en equivalentes disueltos, en ambas lagunas que es:

$\mathrm{Cl} \mathrm{SO} 4$ Alc. $\mathrm{NO}$ ₹ y $\mathrm{Na}^{+} \mathrm{Mg}^{++} \mathrm{Ca}^{++} \mathrm{K}+$

\section{BIBLIOGRAFÍA}

APHA. 1976. Standard Methods for the Examination of Water and Wastewater. APHA, New York. 14th ed. 769 pp.

BREZONICK, P.L. 1972. Nitrogen: sources and transformations in natural waters. In: Nutrient in natural waters (Allen H.E. \& Kramer, J.R. eds.) 1-50. John Wiley \& Sons. New York.

DEL RÍO, M.T. 1986. El ecosistema acuático de las Albuferas de Adra (Almería). Estudio pormenorizado de sus componentes. Tesis Doctoral. Universidad de Granada. 
GOLTERMAN, H.L. 1969. Methods for chemical analysis of fresh-water. I.B.P. Handbook ng 8. Blackwell. Oxford.

I.G.M.E. 1977. Estudio hidroldgico de la cuenca Sur: Almeria. Informe Técnico IX. Adra.

LOPEZ, P. 1983. Aguas salinas epicontenentales próximas a la costa mediterránea española. Estudio del medio. Tesis Doctoral. Universidad de Barcelona.

MIRACLE, R.M. 1977. Ecosistemas valencianos: Las zonas humedas litorales. En: E1 medio ambiente en la comunidad valenciana (Generalitat Valenciana ed.): 76-81. Valencia.

ROS, J.D. 1979. Prácticas de Ecología. Omega. Barcelona. 181 p.
SÁNCHEZ CASTILLO, P. 1984. Estudio limnológico de dos ecosistemas hidricos cle In Provincia de Almería. Excma. Diputación Provincial de Almeria. Almeria.

SANCHEZ CASTILLO, P.; P. CARRILLO; J.M. CRUZ MARTINEZ y L. CRUZ PIZARRO. 1987. Plancton de las lagunas de la Albufera de Adra: Ciclo anual. I.E.A. Excma. Diputación Provincial de Almeria. Almería. (en prensa).

SERRA, M.; M.R. MIRACLE y E. VICENTE. 1984. Interrelaciones entre los principales parámetros limnológicos de la Albufera de Valencia. Limnetica 1:9-19. 